\title{
Estratégias metodológicas para elaboração de material educativo: em foco a promoção do desenvolvimento de prematuros
}

\author{
Methodological strategies for the elaboration of educational \\ material: focus on the promotion of preterm infants' development
}

Rayla Amaral Lemos (https://orcid.org/0000-0003-3090-1806) ${ }^{1}$

Maria de La Ó Ramallo Veríssimo (https://orcid.org/0000-0002-5474-0245) ${ }^{2}$
${ }^{1}$ Faculdade de Fisioterapia, Universidade Federal de Juiz de Fora. R. Eugênio do Nascimento s/n, Bairro Dom Bosco. 36038-330 Juiz de Fora MG Brasil. rayla.lemos@uff.edu.br ${ }^{2}$ Departamento de Enfermagem MaternoInfantil e Psiquiátrica, Escola de Enfermagem, Universidade de São Paulo. São Paulo SP Brasil.

\begin{abstract}
This study aimed to describe and analyze the process of elaborating educational material to promote the development of preterm infants. The procedures included participative exploratory research with the target audience, integrative reviews of the literature on the subject, and systematization of contents through robust theoretical references. Popular Education in Health guided the elaboration of the educational material, and the participatory approach; the Bioecological Theory of Human Development conducted the analysis and systematization of the contents; and Simply Put guided the graphic organization of the material. The theoretical model of the development of preterm infants, and data systematization and organization, allowed the elaboration of an interactive educational material, a technology in a book format for the family. The multiple research strategies and theoretical references have provided methodological rigor, increasing the educational material's action potential, which draws family experience closer to the updated scientific knowledge, thus enhancing child health promotion.
\end{abstract}

Key words Health education, Child development, Premature Infant, Educational and Promotional Materials, Educational Technology
Resumo O objetivo deste estudo foi descrever e analisar o processo de elaboração de um material educativo para a promoção do desenvolvimento da criança nascida prematura. Os procedimentos incluíram: pesquisa exploratória participativa com o público destinatário, revisões integrativas de literatura sobre o tema e sistematização dos conteúdos por meio de referenciais teóricos robustos. A Educação Popular em Saúde orientou a elaboração do material educativo e a abordagem participativa, a Teoria Bioecológica do Desenvolvimento Humano balizou a análise e sistematização dos conteúdos, e o Simply Put a organização gráfica do material. O modelo teórico do desenvolvimento da criança nascida prematura e a sistematização e organização dos dados propiciou a elaboração de um material educativo interativo, uma tecnologia em formato de livro destinada à família. As múltiplas estratégias de pesquisa e os referenciais teóricos conferiram rigor metodológico, ampliando o potencial de ação do material educativo, que aproxima a experiência familiar ao conhecimento científico atualizado potencializando a promoção da saúde da criança.

Palavras-chave Educação em saúde, Desenvolvimento infantil, Recém-Nascido Prematuro, Materiais Educativos e de Divulgação, Tecnologia educacional 


\section{Introdução}

Este artigo apresenta o percurso metodológico, os referenciais e as estratégias de pesquisa utilizadas na elaboração do material educativo "História de Sofia: batalhas e conquistas da família no cuidado e desenvolvimento da criança prematura", que busca amparar uma relevante questão de saúde coletiva: a promoção do desenvolvimento em condição de prematuridade.

A prematuridade é um importante problema de saúde mundial em virtude do crescente número de nascimentos nesta condição $0^{1-3}$ e por ser a precursora de inúmeras morbidades e prejuízos no desenvolvimento infantil, que podem repercutir ao longo da vida ${ }^{4-7}$. Destacam-se prejuízos no desenvolvimento funcional, que afetam a capacidade de execução de atividades de vida diária e exercício do papel social esperado para faixa etária, significantemente prevalentes na população de nascidos prematuros ${ }^{5,6}$.

O guia nacional de orientações para profissionais que atendem crianças nascidas prematuras reforça que a intervenção deve contemplar orientação à família, enfatizando-a como parceria no cuidado e desenvolvimento da criançå ${ }^{8}$. No entanto, materiais educativos sistematizados para orientação e suporte familiar são escassos, especialmente a este público que apresenta necessidades específicas de orientação.

A falta de sistematização das orientações à família é ressaltada por Chiodi et al. ${ }^{9}$ como uma preocupação de cuidadores e profissionais, destacando que os materiais educativos são ferramentas úteis que poderiam ser disponibilizadas aos pais para instrução e consulta domiciliar quando necessário. Também já foi ressaltada a importância de pesquisas que se dedicaram à elaboração de materiais educativos para orientação da família ${ }^{10}$.

O Ministério da Saúde (MS) do Brasil, por meio da política Nacional de Educação Popular e Saúde ${ }^{11}$, e de seus referenciais, $\mathrm{I}^{12}$ e $\mathrm{II}^{13}$ Cadernos de Educação Popular e Saúde ${ }^{12,13}$, reconhece as ações educativas como importantes e fundamentais estratégias para enfrentamento das questões de saúde pública e promoção da saúde. A revisão sistemática de Silva et al. ${ }^{14}$ mostrou o impacto positivo de estratégias educativas participativas, como a utilização de materiais educativos durante visitas domiciliares, no enfrentamento de importantes questões de saúde, gerando desfechos favoráveis como a redução dos índices de prematuridade e baixo peso ao nascimento e aumento do aleitamento materno exclusivo. Desfecho similar foi também observado no estudo de San- tana et al. ${ }^{15}$. Estes resultados favoráveis das intervenções educativas no empoderamento da família para a melhoria do cuidado e potencialização do desenvolvimento da criança nascida prematura são mundialmente reconhecidos como boas estratégias de promoção da saúde².

Materiais educativos constituem uma tecnologia de cuidado ${ }^{16}$ que potencializa as intervenções de saúde e o trabalho da equipe, pois, além de mediar de maneira lúdica o processo de $\mathrm{em}$ poderamento dos sujeitos para promoção de sua saúde, são ferramentas permanentes de cuidado, uma vez que podem ser consultadas sempre que necessário.

Existem muitos estudos e publicações dedicados à explanação metodológica da construção e validação de instrumentos de medida ${ }^{17,18}$, bem como de apresentação de pesquisas dedicadas à elaboração e validação de instrumentos padronizados de avaliação do desenvolvimento infantil ${ }^{19}$, incluindo-se os destinados às crianças nascidas prematuras. Porém, são escassos os destinados à orientação da construção metodológica de materiais educativos, especialmente aqueles destinados à orientação e empoderamento da família para a promoção do desenvolvimento infantil, apesar de seu reconhecido e expressivo papel nos resultados positivos na saúde da criança quando devidamente orientada ${ }^{2,9,14,15}$.

Nos primeiros anos de vida da criança nascida prematura, há grande preocupação com aspectos fisiológicos e do crescimento, seja por parte das famílias, seja por parte dos profissionais. O desenvolvimento é, muitas vezes, entendido como sinônimo de crescimento, considerado adequado quando há aumento de peso ou estatura, por exemplo, em detrimento da percepção das aquisições de habilidades funcionais ${ }^{6}$. Além disso, existe uma tendência à superproteção $\mathrm{e}$ limitação da independência da criança nascida prematura $^{6}$. O efeito cumulativo dos aspectos biológicos, sociais e atitudes de cuidado sobre o desenvolvimento da criança, muitas vezes, não são percebidos ${ }^{6}$. Todos estes fatores reforçam a necessidade de orientação sistematizada, abrangente, focada no desenvolvimento e utilizando-se tecnologias educativas adequadas e acessíveis.

Para que os materiais educativos sejam apropriados ao público a qual se destinam, e ao constructo a ser veiculado e trabalhado, devem ser construídos por meio de bases metodológicas robustas, com estratégias de construção válidas e confiáveis, e referenciais teóricos adequados ${ }^{12,13}$. Além disso, devem contar com a participação efetiva de membros do grupo destinatário ${ }^{20-22}$. 
Diante da reconhecida importância dos materiais educativos na saúde da população infantil, coloca-se a questão: Como elaborar materiais educativos adequados e representativos das necessidades da população a qual se destina, com base na educação popular?

Assim, o objetivo deste estudo foi descrever e analisar o processo metodológico de elaboração de um material educativo voltado para a promoção do desenvolvimento funcional de crianças nascidas prematuras.

\section{Métodos}

Estudo metodológico com foco na descrição e análise do processo de elaboração de um material educativo para promoção do desenvolvimento da criança nascida prematura, orientado pelos referenciais da educação popular e saúde e da teoria bioecológica do desenvolvimento humano. Este estudo é parte de uma pesquisa maior e respeitou todos os preceitos éticos, com aprovação pelo Comitê de Ética em Pesquisa, em outubro de 2013 .

\section{Referenciais teórico e metodológico}

O referencial da Educação Popular e Saú$\mathrm{de}^{11-13}$ sistematizado por Paulo Freire orientou a elaboração do material educativo, no que diz respeito a suas etapas e estratégias metodológicas, e a Teoria Bioecológica do Desenvolvimento Humano $^{23}$ balizou a análise e sistematização dos conteúdos nele abordados.

A educação Popular preconiza o método participativo como premissa para o empoderamento e autonomia dos sujeitos para cuidado de sua saúde. Esta metodologia emancipadora leva em conta o entendimento e a experiência da pessoa que vive no mundo e atua na transformação de sua realidade. Propõe que este entendimento, muitas vezes, fragmentado e pouco elaborado, seja o objeto de ação da educação popular em saúde, mediante discussões de forma horizontal e problematizada, de acordo com as situações vividas pelos sujeitos e comunidades ${ }^{12,13}$.

A Teoria bioecológica do desenvolvimento humano ${ }^{23}$ concebe o desenvolvimento humano ancorado e direcionado pelos componentes: Processo, Pessoa, Contexto e Tempo.

O Processo, colocado como principal eixo do desenvolvimento, refere-se às interações recíprocas, sistemáticas, estabelecidas por longo período de tempo entre o sujeito em desenvolvimento e as pessoas, objetos e símbolos presentes em seu ambiente próximo ${ }^{23}$. A Pessoa é o indivíduo em desenvolvimento com suas características individuais, genéticas, psicossociais e comportamentais, que atua no ambiente e em seu próprio desenvolvimento e também gera respostas do Contexto em que está inserida ${ }^{23}$. O Contexto é o ambiente em que a Pessoa desenvolve. Divide-se em: Microssistema, ambiente próximo em que o sujeito está inserido e no qual estabelece interações diretas; Mesossistema, referente à relação entre os microssistemas; Exossistema, ambiente em que a Pessoa não está diretamente inserida, mas que influencia seu desenvolvimento; e Macrossistema, conjunto de valores, ideologia e crenças que permeiam o ambiente da Pessoa ${ }^{23}$. O Tempo é o elemento relacionado às mudanças e estabilidades do desenvolvimento da Pessoa ao longo do tempo, e também ao tempo histórico em que vive ${ }^{24}$. A Figura 1 apresenta o modelo esquemático da teoria bioecológica do desenvolvimento humano, com os componentes $\mathrm{PPCT}^{24}$.

\section{Percurso metodológico}

O percurso metodológico para identificação do conteúdo e composição do material educativo seguiu os preceitos da construção de instrumentos de medida na área de saúde ${ }^{18}$, adaptados para elaboração de um material educativo, excluindose apenas os aspectos específicos de instrumentos de medida.

O Quadro 1 sumariza os objetivos de cada etapa para composição do material educativo, os recursos utilizados para o alcance de cada etapa e as definições estabelecidas na organização do material.

Após estabelecimento dos recursos e definições para composição do material educativo, prosseguiu-se definindo e aplicando estratégias para seleção, organização e estruturação dos conteúdos e ilustrações do material.

A primeira estratégia desenvolvida foi um estudo exploratório com representantes da população alvo. Por meio de entrevistas semiestruturadas com 12 cuidadores de crianças nascidas prematuras, acompanhadas em serviço de referência para atendimento desta população, verificou-se a compreensão dos cuidadores acerca do desenvolvimento de suas crianças, suas necessidades, dúvidas e componentes do cuidado para promoção do desenvolvimento da criança. As entrevistas foram conduzidas de forma participativa e dialógica, utilizando roteiro estruturado e estratégias lúdicas, como quadros ilustrativos. Buscaram ex- 


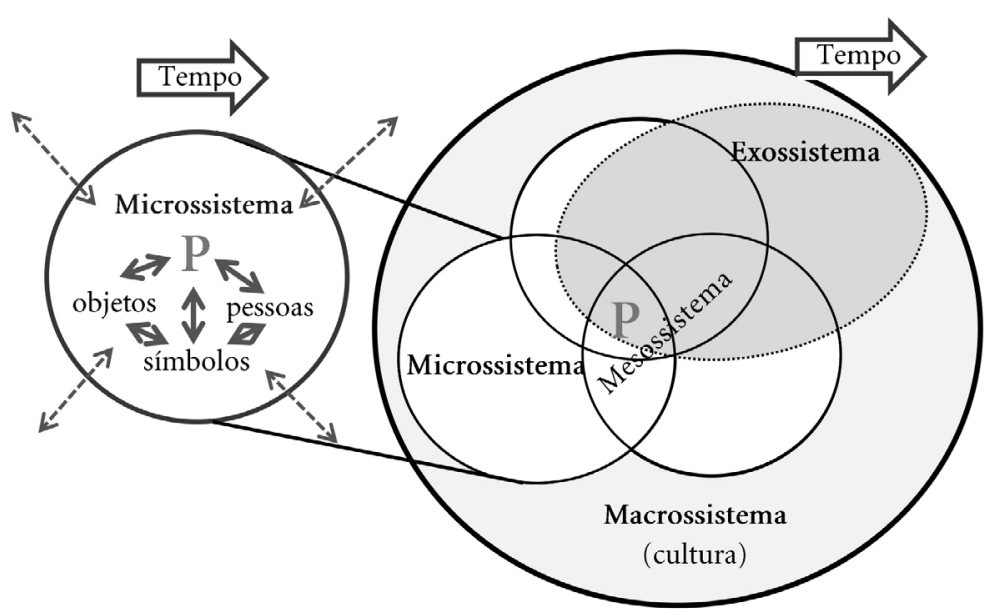

Figura 1. Modelo PPCT.

Notas: A Pessoa (P) ativa engajada em Processos Proximais com pessoas, símbolos e objetos dentro de um microssistema, em interação com outros Contextos, incluindo tanto a continuidade quanto a mudança ao longo do Tempo. Fonte: Tudge ${ }^{24}$.

plorar a experiência vivida pelos cuidadores e as questões que dela emergiram. Realizou-se análise qualitativa de conteúdo dos dados seguindo o referencial da Teoria Bioecológica do Desenvolvimento Humano ${ }^{23}$.

A segunda estratégia estabelecida para elaboração do material educativo foi revisão de literatura. Foram realizadas duas revisões integrativas de literatura sobre práticas de cuidado promotoras do desenvolvimento funcional no domicílio cujas questões norteadoras foram: "Quais são as práticas de cuidado que promovem o desenvolvimento funcional de nascidos prematuros até os três anos de idade?" e "Quais fatores/elementos estão envolvidos no cuidado prestado pela família à criança nascida prematura após a saída da UTIN?" As revisões seguiram os passos da Revisão Integrativa ${ }^{25}$ e ocorreram nos meses de janeiro/fevereiro e agosto/setembro de 2015 respectivamente.

Para a primeira revisão integrativa, realizaram-se buscas nas bases Science Direct, Scopus, e Web of Science com os seguintes descritores e suas combinações, em língua inglesa e portuguesa: preterm, child care e development. Na segunda revisão, foram incluídas as bases de dados Literatura Latino-Americana e do Caribe em Ciências da Saúde - Lilacs, PsicoInfo [APA, PsycNet], Scientific Electronic Library Online - SciELO,
Physiotherapy Evidence Database - PEDro, Biblioteca Virtual de Saúde - BVS, Institute of Education Sciences - Eric. Foram incluídos também outros descritores e suas combinações na língua inglesa e portuguesa: preterm infant, development promotion, health education, Family education, nurture intervention e health promotion.

Os critérios de inclusão foram: artigos completos em língua inglesa ou portuguesa, publicados nos periódicos indexados nas referidas bases de dados nos últimos cinco anos. Foram excluídos artigos que investigaram faixas etárias diferentes da preconizada para o material educativo e aqueles com temáticas não condizentes com as questões norteadoras.

Após leitura do título e do resumo, foram selecionados os artigos que foram lidos na íntegra e organizados em uma tabela de análise com dados de identificação, autoria, ano de publicação, tipo de estudo, sujeitos da pesquisa, objetivo/avaliação e os principais resultados. Nessa etapa, foram excluídos os artigos que, após a leitura integral, não atenderam os critérios de inclusão.

Após a sistematização dos conteúdos, optouse por utilizar também livros-texto, artigos, documentos e Lei, considerados referências-chave, por seu conteúdo pertinente e relevante para a constituição das bases teóricas e operacionais da sistematização do material educativo. Estes 
Quadro 1. Descrição das etapas, seus objetivos, recursos e resultados para composição do material educativo sobre promoção do desenvolvimento da criança prematura.

\begin{tabular}{|c|c|c|c|}
\hline Etapa preconizada* & Objetivo da etapa & Recursos utilizados & Resultados/Definições \\
\hline $\begin{array}{l}\text { Estabelecimento da } \\
\text { estrutura conceitual }\end{array}$ & $\begin{array}{l}\text { Dimensionar e } \\
\text { definir o contexto } \\
\text { do instrumento } \\
\text { para sustentar cada } \\
\text { conteúdo nele } \\
\text { inserido. }\end{array}$ & $\begin{array}{l}\text { Revisão de literatura; } \\
\text { Experiência profissional } \\
\text { das pesquisadoras; } \\
\text { Pesquisa com população } \\
\text { alvo. }\end{array}$ & $\begin{array}{l}\text { Referenciais selecionados: } \\
\text { Promoção do desenvolvimento; } \\
\text { Desenvolvimento funcional; } \\
\text { Educação Popular e Saúde; } \\
\text { Bioecologia do desenvolvimento } \\
\text { humano. }\end{array}$ \\
\hline $\begin{array}{l}\text { Definição de } \\
\text { objetivos }\end{array}$ & $\begin{array}{l}\text { Estabelecer a } \\
\text { proficuidade do } \\
\text { material. }\end{array}$ & $\begin{array}{l}\text { Articulação entre as } \\
\text { necessidades da população } \\
\text { com os referenciais } \\
\text { teóricos adotados. }\end{array}$ & $\begin{array}{l}\text { Objetivo do material: } \\
\text { Apoiar a família para o cuidado e } \\
\text { promoção do desenvolvimento da } \\
\text { criança nascida prematura. }\end{array}$ \\
\hline $\begin{array}{l}\text { Definição da } \\
\text { População Alvo }\end{array}$ & $\begin{array}{l}\text { Estabelecer a } \\
\text { proficuidade do } \\
\text { material e justificar } \\
\text { a relevância do } \\
\text { material produzido. }\end{array}$ & $\begin{array}{l}\text { Revisão de Literatura; } \\
\text { Experiência profissional } \\
\text { das pesquisadoras; } \\
\text { Relatos da população alvo. }\end{array}$ & $\begin{array}{l}\text { População alvo: } \\
\text { Cuidadores de crianças nascidas } \\
\text { prematuras com idade atual inferior } \\
\text { a três anos. }\end{array}$ \\
\hline $\begin{array}{l}\text { Elaboração dos } \\
\text { itens: } \\
\text { Seleção, } \\
\text { Organização e } \\
\text { Estruturação dos } \\
\text { conteúdos do } \\
\text { material - textos e } \\
\text { ilustrações }\end{array}$ & $\begin{array}{l}\text { Estabelecer a } \\
\text { proficuidade do } \\
\text { material condizente } \\
\text { com os referenciais } \\
\text { estabelecidos. }\end{array}$ & $\begin{array}{l}\text { Revisão de literatura; } \\
\text { Análise de materiais } \\
\text { existentes sobre o tema; } \\
\text { Pesquisa com a população- } \\
\text { alvo; } \\
\text { Observação clínica; } \\
\text { Opinião de especialistas. }\end{array}$ & $\begin{array}{l}\text { Características do material: } \\
\text { Material dialogado e interativo; } \\
\text { Em formato de história, dividida em } \\
\text { capítulos, com conteúdos definidos } \\
\text { de acordo com as necessidades da } \\
\text { população alvo e organizados com } \\
\text { linearidade na história; } \\
\text { Ilustrações definidas em } \\
\text { conformidade com os conteúdos; } \\
\text { Inclusão de encarte para } \\
\text { acompanhamento do } \\
\text { desenvolvimento funcional. }\end{array}$ \\
\hline
\end{tabular}

* Referem-se às etapas 1 e 2 preconizadas pelos autores Coluci et al. ${ }^{18}$ relativas à elaboração do material educativo; a etapa 3 , validação de conteúdo e de face com juízes e população alvo, será tratada em outro texto.

consistiram, basicamente, em textos sobre o referencial teórico de análise e o referencial metodológico de construção de materiais educativos, conteúdos esses ausentes ou não detalhados nos artigos da revisão.

A terceira estratégia metodológica foi a busca de referenciais que orientassem a elaboração operacional das partes de um material educativo destinado à população. O referencial selecionado e utilizado para a elaboração do material educativo em tela foi o Simply Put - A guide for creating easy-to-understand materials, elaborado pelo Center of Diseases Control and Prevention do Departamento de Saúde e Serviços Humanos dos EUA $^{26}$. Ele fornece estratégias para construção de materiais educativos em saúde mais compreensíveis, especialmente para populações com baixa escolaridade, apontando recomendações para todo o processo de constituição, incluindo aspectos do conteúdo, da linguagem utilizada, da forma e layout gráficos de textos e imagens, dos aspectos visuais, jogo de cores, tipos de letra, entre outros, como foco no constructo veiculado e tipo de informação a ser trabalhada, bem como ação esperada do sujeito em decorrência da utilização do material na prática.

A utilização das estratégias metodológicas e dos referenciais teóricos adotados orientou a elaboração do material educativo de forma sistemática e com rigor metodológico, como se descreve a seguir. $\mathrm{O}$ material depois de elaborado foi encaminhado para avaliação e validação por profissionais e público destinatário, este conteúdo será tratado em outro artigo, dado não ser objeto deste.

\section{Resultados}

Adicionalmente à análise descritiva apresentada na publicação do estudo exploratório com as 
famílias, a totalidade dos conteúdos desta etapa foi organizada em um banco de dados contendo aspectos da experiência da família, da compreensão sobre o desenvolvimento das crianças, e das necessidades apontadas pelos cuidadores, para, juntamente com os resultados da revisão bibliográfica, serem trabalhadas como conteúdo do material educativo proposto.

Na revisão, foram selecionados 76 artigos, e excluídos 49 , por não se adequarem aos critérios de inclusão estabelecidos. Assim, 27 artigos completos foram incluídos para estudo e composição do material educativo. A maior parte dos estudos foi retirado da base Science Direct (8), seguido da Web of Science (7), Scielo (5), Scopus (5) e PsycoInfo-APA (2).

A maioria dos 27 estudos incluídos estava no idioma inglês (23) e datou do ano de 2014 (16). Os tipos de estudo variaram: caso-controle (6), descritivos (11), de coorte (6), revisão sistemática (2) ou crítica (1), e survey (1). A referência completa e os principais resultados de cada artigo selecionado foram compilados em um quadro síntese que sumarizava seus principais achados e contribuições. Esta organização permitiu identificar, selecionar e sistematizar os conteúdos específicos a serem incluídos no material educativo proposto.

Os textos e fontes de pesquisa e base teórica considerados como referência chave na constituição do material educativo estavam relacionados ao referencial teórico da teoria bioecológica do desenvolvimento humano, ao referencial do desenvolvimento funcional, à prematuridade, $\mathrm{e}$ aos direitos de crianças e adolescentes no Brasil.

A compreensão da prematuridade a partir do Referencial teórico da Teoria Bioecológica do Desenvolvimento Humano como norteadora do material educativo, o estudo exploratório com as famílias na primeira etapa e a revisão de literatura possibilitaram a elaboração de um modelo teórico para compreensão do modelo $\mathrm{PPCT}^{22}$ para a criança nascida prematura, expresso na Figura 2, que balizou o conteúdo do material educativo:

Os conteúdos dos artigos e documentos selecionados para o estudo foram sistematizados em dois quadros de evidência, de acordo com o Referencial do Modelo bioecológico do desenvolvimento humano ${ }^{23} \mathrm{e}$ as dimensões do desenvolvimento funcional ${ }^{27,28}$. Nos Quadros 2 e 3, as categorias estabelecidas estão elencadas com as referências bibliográficas que as originaram, e de acordo dos referenciais teóricos correspondentes.

Essa sistematização possibilitou que todos os conteúdos fossem, então, organizados para com- por o material educativo, pois foram distribuídos integralmente em agrupamentos que compuseram os capítulos do material em forma de livro. A seguir, realizou-se a redação dos capítulos, utilizando-se estratégias para a elaboração de um texto atraente e de linguagem acessível, de acordo com os critérios do Simply Put ${ }^{26}$ e o referencial da Educação Popular e Saúde ${ }^{12,13}$.

Assim, os dados sistematizados foram trabalhados no formato de uma história que retrata as experiências de uma família que viveu a condição do nascimento de uma criança prematura e dialoga com o leitor, indicando informações importantes para o cuidado da criança e a promoção do desenvolvimento funcional. No decorrer do texto, foram delineados espaços interativos para o leitor preencher com suas experiências. Foi também elaborado um quadro de habilidades funcionais esperadas por faixa etária, incluído ao final como um encarte, possibilitando a família acompanhar o desenvolvimento da criança por área do desenvolvimento funcional e idade.

$\mathrm{O}$ material em arquivo de texto simples foi formatado com uma estrutura que incluiu: indicações de capítulos; destaques no texto, com negritos ou caixas de texto coloridas, referentes a informações importantes oriundas da pesquisa e revisão de literatura; indicações dos espaços interativos com o leitor; e descrições da forma, local e tipo de ilustração a ser incluída em cada trecho do material e do encarte. Esta primeira versão foi encaminhada para uma empresa profissional especializada para diagramação e ilustração, contratada com recurso obtido mediante processo de fomento à pesquisa.

Durante o processo de diagramação e ilustração, o material foi periodicamente revisado pelas pesquisadoras em relação ao layout e forma de veiculação das informações em contatos diretos com a empresa. Isto visou potencializar a inclusão de ilustrações que assegurassem informações adequadas ao referencial teórico empregado e aos preceitos estabelecidos pelas instituições de saúde oficiais no Brasil e no mundo.

A primeira versão do material diagramada e ilustrada com 55 páginas foi disponibilizada para o processo de validação de conteúdo e de face com juízes e público alvo. Ainda não continha as referências bibliográficas nem os relatos das famílias, previstos como parte do conteúdo final, apenas indicação de que seriam incluídos. Após o processo de validação, o material foi revisado, ajustado e encaminhado para impressão. O material impresso foi encaminhado para distribuição nos serviços de saúde participantes da 


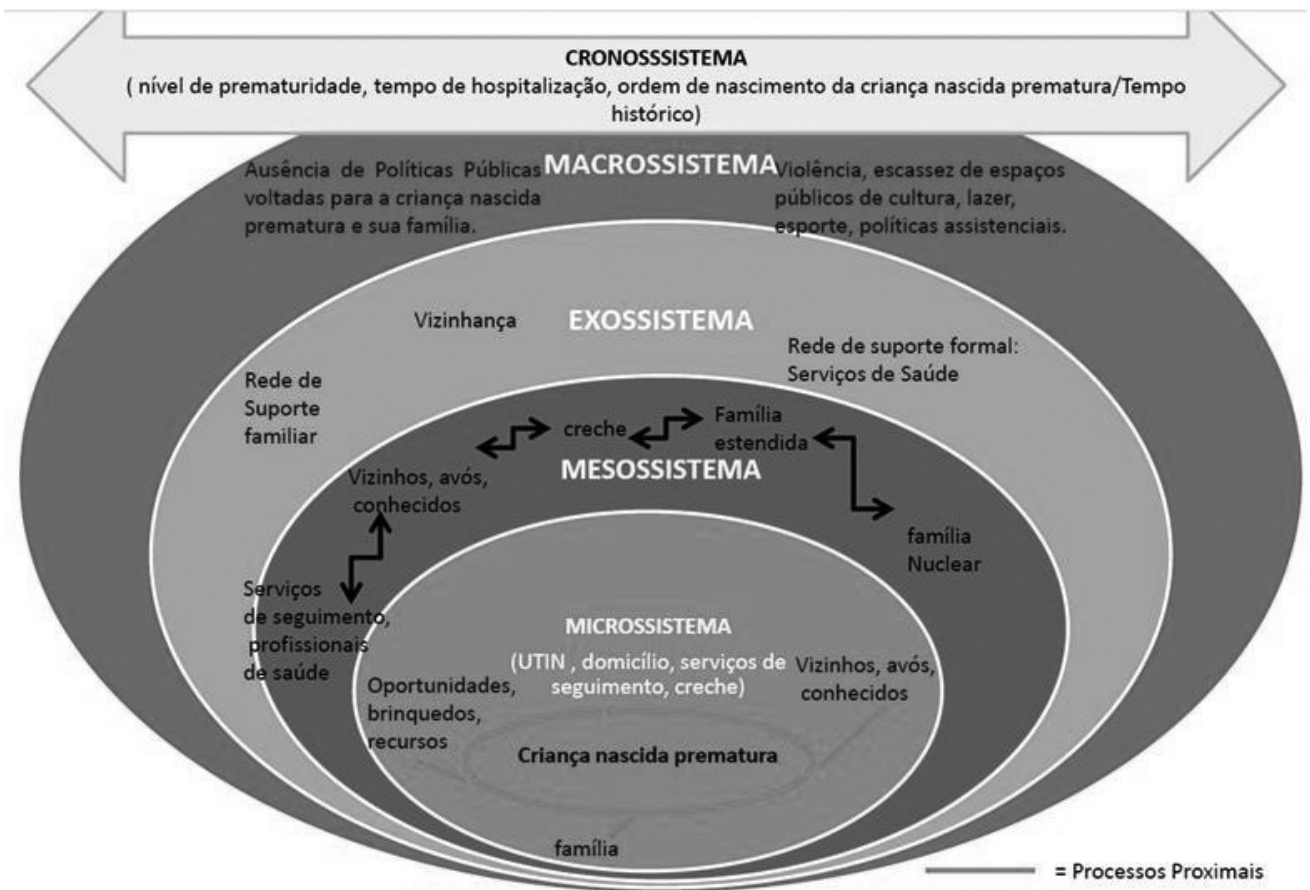

Figura 2. Modelo PPCT para a criança nascida prematura.

Fonte: Elaborada pelas pesquisadoras.

pesquisa e em serviços em que foram estabelecidas continuações da pesquisa. Além disso, foi veiculado no site da instituição de ensino em que a pesquisa foi desenvolvida e foi oferecida sua disponibilização para sites oficiais de associação de cuidadores de nascidos prematuros e para o Ministério da Saúde.

\section{Discussão}

Este estudo buscou contribuir com proposições de construção de materiais educativos, ao descrever e justificar as estratégias metodológicas do processo de elaboração de um material educativo voltado para a promoção do desenvolvimento funcional de crianças nascidas prematuras.

Streck $^{22}$ adverte para necessidade de buscar critérios abertos e consistentes para a pesquisa com o referencial da Educação Popular e com metodologias participativas, seguidos neste estudo: a relevância social, a qualidade de descrição e de interpretação, a reflexividade, a qualidade da relação entre os sujeitos e a praticabilidade do conhecimento.

Ademais, foram seguidas recomendações de estudiosos relativas à construção e validação de instrumentos ${ }^{17,18}$ e de pesquisadores que elaboraram e validaram tecnologias educativas, utilizando métodos similares, para múltiplos fins ${ }^{29,30}$, para área de saúde da criança ${ }^{31,32}$ e para apoio à família de nascidos prematuros ${ }^{33}$.

A utilização do modelo PPCT da Teoria Bioecológica do Desenvolvimento Humano ${ }^{23}$ na sistematização dos resultados da pesquisa com as famílias de crianças nascidas prematuras ${ }^{34}$ e da revisão de literatura, foi coerente com o objetivo de apoiar a família mediante criação do material educativo para o cuidado e promoção do desenvolvimento da criança nascida prematura. Isto porque a promoção do desenvolvimento da criança é um efeito dos Processos Proximais estabelecidos entre as Pessoas que interagem nos Contextos e no $\mathrm{Tempo}^{23}$. Assim, os processos de interação 
Quadro 2. Aspectos relacionados ao cuidado da criança nascida prematura e suas fontes bibliográficas, segundo o modelo PPCT.

\begin{tabular}{|c|c|c|}
\hline \multirow[t]{2}{*}{$\begin{array}{l}\text { Classificação } \\
\text { PPCT }\end{array}$} & Aspecto Relacionado ao Cuidado & Referência \\
\hline & \multicolumn{2}{|c|}{ Fatores de influência positiva sobre o desenvolvimento de crianças nascidas prematuras } \\
\hline \multirow[t]{19}{*}{ Processo } & $\begin{array}{l}\text { Afeto, interação e presença contínua e participativa dos } \\
\text { cuidadores. }\end{array}$ & $\begin{array}{l}\text { Lemos e Veríssimo, } 2015^{34} \\
\text { Lundqvist et al., } 2014^{35}\end{array}$ \\
\hline & Brincadeiras, brinquedos e conversas. & $\begin{array}{l}\text { Lemos e Veríssimo, } 2015^{34} \\
\text { Hall et al., } 2013^{36}\end{array}$ \\
\hline & Estimulação da criança e ensino de habilidades. & Lemos e Veríssimo, $2015^{34}$ \\
\hline & Leitura, música ou esporte. & Lemos e Veríssimo, $2015^{34}$ \\
\hline & Oferecimento de alimentos saudáveis. & Lemos e Veríssimo, $2015^{34}$ \\
\hline & $\begin{array}{l}\text { Convívio com outras crianças em casa, na vizinhança, ou } \\
\text { na creche. }\end{array}$ & Lemos e Veríssimo, $2015^{34}$ \\
\hline & Cuidado baseado nas pistas oferecidas pela criança. & Evans et al., $2014^{37}$ \\
\hline & $\begin{array}{l}\text { Ouvir a voz materna; } \\
\text { Interações e verbalizações com o RN ainda na UTIN; } \\
\text { Estimulação multissensorial (posicionamento, massagem, } \\
\text { estimulação oral...) oferecido ainda na UTIN pelos } \\
\text { cuidadores treinados pela equipe. }\end{array}$ & $\begin{array}{l}\text { Picciolini et al., } 20144 \\
\text { Caskey et al., } 201138 \\
\text { Gerstein et al., } 201539 \\
\text { Gabis et al., } 201540\end{array}$ \\
\hline & $\begin{array}{l}\text { Perceber a criança se desenvolvendo, a aquisição de } \\
\text { habilidades traz alívio aos cuidadores. }\end{array}$ & $\begin{array}{l}\text { Lemos e Veríssimo, } 2015^{34} \\
\text { Lundqvist et al., } 2014^{35}\end{array}$ \\
\hline & \multicolumn{2}{|c|}{ Fatores de influência negativa sobre o desenvolvimento de crianças nascidas prematuras } \\
\hline & Maus-tratos e ausência de afeto ou carinho dos cuidadores. & Lemos e Veríssimo, $2015^{34}$ \\
\hline & $\begin{array}{l}\text { Expectativa demasiada e apressada sobre a trajetória de } \\
\text { desenvolvimento e/ ou comparação do desenvolvimento } \\
\text { com o de outras crianças. }\end{array}$ & $\begin{array}{l}\text { Lemos e Veríssimo, } 2015^{34} \\
\text { Lundqvist et al., } 2014^{35} \\
\text { Hall et al., } 2013^{36}\end{array}$ \\
\hline & $\begin{array}{l}\text { Cuidado restritivo à independência da criança e } \\
\text { superproteção. }\end{array}$ & $\begin{array}{l}\text { Lemos e Veríssimo, } 2015^{34} \\
\text { Whittingham et al., } 2014^{41} \\
\text { Morais et al., } 2009^{42} \\
\text { Souza et al., } 2010^{25}\end{array}$ \\
\hline & $\begin{array}{l}\text { Fala, pensamento ou influência (negativa) de pessoas } \\
\text { próximas ou externas ao convívio sobre a criança e seu } \\
\text { desenvolvimento. }\end{array}$ & $\begin{array}{l}\text { Lemos e Veríssimo, } 2015^{34} \\
\text { Souza et al., } 2010^{25} \\
\text { McGowan et al., } 2014^{43} \\
\text { Lundqvist et al., } 2014^{35} \\
\text { Pal et al., 2014 }\end{array}$ \\
\hline & $\begin{array}{l}\text { Preocupação com o desenvolvimento atual e futuro } \\
\text { (intercorrências/ deficiências, atrasos, efeitos colaterais) e } \\
\text { estado de saúde atual, ou futuro. }\end{array}$ & $\begin{array}{l}\text { Lemos e Veríssimo, } 2015^{34} \\
\text { McGowan et al., } 2014^{43} \\
\text { Lundqvist et al., } 2014^{35} \\
\text { Hall et al., } 2013^{36} \\
\text { Pal et al., 2014 }\end{array}$ \\
\hline & $\begin{array}{l}\text { Cuidadores às vezes colocam seu autocuidado em } \\
\text { segundo plano. }\end{array}$ & Hall et al., $2013^{36}$ \\
\hline & $\begin{array}{l}\text { Cuidadores perceberem pouco as pistas dadas pela criança } \\
\text { durante o cuidado. }\end{array}$ & Winstanley et al., $2014^{45}$ \\
\hline & $\begin{array}{l}\text { A experiência do período inicial é traumática, traz medo, } \\
\text { inseguranças, mesmo depois de percebida como acabada. }\end{array}$ & $\begin{array}{l}\text { Lemos e Veríssimo, } 2015^{34} \\
\text { Lundqvist et al., 2014 } \\
\text { Pal et al., } 2014^{44} \\
\end{array}$ \\
\hline & $\begin{array}{l}\text { Problemas de saúde mental: estresse, ansiedade, depressão, } \\
\text { estresse pós-traumático; } \\
\text { Sintomas de depressão materna, estresse dos cuidadores é } \\
\text { fator de risco para o desenvolvimento social, emocional e } \\
\text { funcional de nascidos prematuros aos } 5 \text { anos de idade. O } \\
\text { senso de coerência das mães pode ser um fator protetor; } \\
\text { Sintomas de depressão materna parecem ter efeitos } \\
\text { negativos sobre a função cognitiva de crianças nascidas } \\
\text { prematuras aos } 16 \text { meses. }\end{array}$ & $\begin{array}{l}\text { Howe et al., } 2014^{46} \\
\text { Gray et al., } 2013^{47} \\
\text { Hall et al., } 2013^{36} \\
\text { Chang et al., } 2014^{48} \\
\text { Mehler et al., } 2014^{49} \\
\text { Suttora et al., 2013 } 20 \\
\text { Huhtala et al., 2014 } \\
\text { McManusa e Poehlmannb, 2012 }\end{array}$ \\
\hline
\end{tabular}


Quadro 2. Aspectos relacionados ao cuidado da criança nascida prematura e suas fontes bibliográficas, segundo o modelo PPCT.

\begin{tabular}{|c|c|c|}
\hline $\begin{array}{l}\text { Classificação } \\
\text { PPCT }\end{array}$ & Aspecto Relacionado ao Cuidado & Referência \\
\hline \multirow[t]{4}{*}{ Pessoa } & $\begin{array}{l}\text { O nascimento prematuro define uma demanda da } \\
\text { criança: pequenas ou frágeis... (Fragilidade). }\end{array}$ & $\begin{array}{l}\text { Lemos e Veríssimo, } 2015^{34} \\
\text { Pal et al., } 2014^{44}\end{array}$ \\
\hline & $\begin{array}{l}\text { As famílias não têm clareza sobre Idade corrigida e Idade } \\
\text { cronológica. }\end{array}$ & Lemos e Veríssimo, $2015^{34}$ \\
\hline & $\begin{array}{l}\text { O ganho de peso e o crescimento são entendidos } \\
\text { como progresso do desenvolvimento se a característica } \\
\text { "aumento de peso" está presente, a família acredita que a } \\
\text { criança está indo bem. }\end{array}$ & Lemos e Veríssimo, $2015^{34}$ \\
\hline & Pais de nascidos prematuros tem alto crescimento pessoal. & Taubman et al., $2014^{53}$ \\
\hline \multirow[t]{11}{*}{ Contexto } & \multicolumn{2}{|c|}{ Microssistema } \\
\hline & $\begin{array}{l}\text { Práticas aprendidas na UTIN são reproduzidas em casa, } \\
\text { institucionalização do cuidado. }\end{array}$ & $\begin{array}{l}\text { Couto e Praça, } 2009^{10} \\
\text { Morais et al., 200942 } \\
\text { Costa et al., 200954 } \\
\text { Whittingham et al., } 2014^{41}\end{array}$ \\
\hline & $\begin{array}{l}\text { Existe uma lacuna no fornecimento de informações } \\
\text { da equipe de saúde ou de materiais educativos para } \\
\text { orientação para preparação dos pais para a alta da criança } \\
\text { da UTIN para domicílio, prejudicando a organização do } \\
\text { ambiente domiciliar para o cuidado. }\end{array}$ & $\begin{array}{l}\text { Lemos e Veríssimo, } 2015^{34} \\
\text { Whittingham et al., } 2014^{41} \\
\text { Souza et al., 2010 } \\
\text { Raffray et al., 2014 } \\
\text { McGowan et al., 2014 } \\
\text { Hall et al., 2013 } \\
\text { Pal et al., 2014 } \\
\end{array}$ \\
\hline & $\begin{array}{l}\text { Conflitos no domicílio e lares sem um dos cônjuges } \\
\text { podem ser prejudiciais ao desenvolvimento, segundo } \\
\text { cuidadores. O apoio do cônjuge é essencial. }\end{array}$ & $\begin{array}{l}\text { Lemos e Veríssimo, } 2015^{34} \\
\text { Lundqvist et al., 2014 }\end{array}$ \\
\hline & $\begin{array}{l}\text { Problemas familiares com o funcionamento físico, } \\
\text { incluindo sensação de cansaço, ter dores de cabeça, } \\
\text { sensação de fraqueza, e problemas de estômago. }\end{array}$ & $\begin{array}{l}\text { McGowan et al., } 2014^{43} \\
\text { Howe et al., 2014 } \\
\text { Lundqvist et al., } 2014^{35}\end{array}$ \\
\hline & $\begin{array}{l}\text { Problemas familiares com o funcionamento social, } \\
\text { incluindo a sensação de isolamento, dificuldade em obter } \\
\text { apoio de outras pessoas, e encontrar tempo ou energia } \\
\text { para atividades sociais. Restrição de seu papel social. }\end{array}$ & $\begin{array}{l}\text { McGowan et al., } 2014^{43} \\
\text { Lundqvist et al., } 2014^{35}\end{array}$ \\
\hline & $\begin{array}{l}\text { Problemas familiares com a comunicação, incluindo } \\
\text { não compreensão da situação da família pelos outros, } \\
\text { dificuldade para falar sobre o estado de saúde da criança, } \\
\text { e comunicação com os profissionais de saúde. }\end{array}$ & McGowan et al., $2014^{43}$ \\
\hline & $\begin{array}{l}\text { Problemas familiares com atividades diárias, incluindo } \\
\text { atividades que exigem mais tempo e esforço, dificuldade } \\
\text { em encontrar tempo e energia para terminar as tarefas } \\
\text { domésticas (Funcionamento familiar). }\end{array}$ & $\begin{array}{l}\text { McGowan et al., } 2014^{43} \\
\text { Lundqvist et al., } 2014^{35}\end{array}$ \\
\hline & $\begin{array}{l}\text { Problemas nas relações familiares, incluindo a } \\
\text { comunicação, estresse e conflitos entre membros da } \\
\text { família, e dificuldade em tomar decisões e resolução de } \\
\text { problemas como uma família (Funcionamento familiar); } \\
\text { Ou problemas para obter apoio do cônjuge. }\end{array}$ & $\begin{array}{l}\text { McGowan et al., } 2014^{43} \\
\text { Howe et al., 2014 } \\
\text { Lundqvist et al., } 2014^{35}\end{array}$ \\
\hline & $\begin{array}{l}\text { A creche e/ou os serviços/profissionais de saúde podem } \\
\text { ser promotores do desenvolvimento e favorecer boas } \\
\text { atitudes de cuidadores. }\end{array}$ & $\begin{array}{l}\text { Lemos e Veríssimo, } 2015^{34} \\
\text { Hall et al., } 2013^{36} \\
\text { Guillaume et al., } 2013^{56}\end{array}$ \\
\hline & $\begin{array}{l}\text { Brinquedos, brincadeiras, contextos desafiadores } \\
\text { favorecem o desenvolvimento. }\end{array}$ & Lemos e Veríssimo, $2015^{34}$ \\
\hline
\end{tabular}




\begin{tabular}{|c|c|c|c|}
\hline \multirow{15}{*}{ 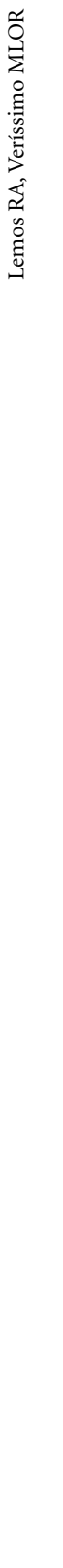 } & \multicolumn{3}{|c|}{$\begin{array}{l}\text { Quadro 2. Aspectos relacionados ao cuidado da criança nascida prematura e suas fontes bibliográficas, segundo } \\
\text { o modelo PPCT. }\end{array}$} \\
\hline & \begin{tabular}{|c|} 
Classificação \\
PPCT
\end{tabular} & Aspecto Relacionado ao Cuidado & Referência \\
\hline & \multirow[t]{11}{*}{ Contexto } & \multicolumn{2}{|l|}{ Mesossistema } \\
\hline & & $\begin{array}{l}\text { A relação entre serviços de saúde em que as crianças } \\
\text { estão inseridas, ou receberam assistência, podem } \\
\text { ser promotores ou limitadores do desenvolvimento } \\
\text { dependendo se esta relação foi positiva ou negativa. }\end{array}$ & $\begin{array}{l}\text { Lemos e Veríssimo, } 2015^{34} \\
\text { Pal et al., } 2014^{44}\end{array}$ \\
\hline & & \multicolumn{2}{|l|}{ Exossistema } \\
\hline & & $\begin{array}{l}\text { Crianças prematuras receberam mais visitas de } \\
\text { profissionais de saúde ou foram mais vezes para consultas e } \\
\text { atendimentos, suporte especializado de serviços de saúde. }\end{array}$ & $\begin{array}{l}\text { Lemos e Veríssimo, } 2015^{34} \\
\text { McGowan et al., } 2014^{43}\end{array}$ \\
\hline & & \multicolumn{2}{|l|}{ Micro e Mesossistemas } \\
\hline & & $\begin{array}{l}\text { A rede de suporte familiar (família, amigos, avós vizinhos) } \\
\text { e/ou assistencial de saúde (serviços e profissionais) é } \\
\text { apontada como crucial para apoiar no cuidado e/ou para } \\
\text { o desenvolvimento da criança. }\end{array}$ & $\begin{array}{l}\text { Lemos e Veríssimo, } 2015^{34} \\
\text { Lundqvist et al., } 2014^{35} \\
\text { Hall et al., 2013 } \\
\text { Chang et al., } 2014^{48} \\
\text { Pal et al., 2014 } \\
\text { Custódio et al., 2014 }\end{array}$ \\
\hline & & \multicolumn{2}{|l|}{ Exossistema } \\
\hline & & $\begin{array}{l}\text { Ausência de espaços de convivência, violência e tráfico } \\
\text { de drogas nos espaços públicos de utilização coletiva e } \\
\text { vizinhança são colocados como exossistemas prejudiciais } \\
\text { ao desenvolvimento. }\end{array}$ & Lemos e Veríssimo, $2015^{34}$ \\
\hline & & $\begin{array}{l}\text { A experiência de outros cuidadores que vivem ou } \\
\text { viveram a mesma condição é vista como positiva e } \\
\text { muito importante pelos cuidadores a se relacionarem e } \\
\text { compartilharem as experiências. }\end{array}$ & $\begin{array}{l}\text { Hall et al., } 2013^{36} \\
\text { Pal et al., } 2014^{44}\end{array}$ \\
\hline & & \multicolumn{2}{|l|}{ Macrossistema } \\
\hline & & $\begin{array}{l}\text { Falta de políticas públicas de assistência social, } \\
\text { cultura e lazer e violência urbana são colocados como } \\
\text { macrossistema prejudicial ao desenvolvimento. }\end{array}$ & Lemos e Veríssimo, $2015^{34}$ \\
\hline & \multirow[t]{2}{*}{ Tempo } & \multicolumn{2}{|l|}{ Micro e Mesotempo } \\
\hline & & $\begin{array}{l}\text { A família reconhece que a criança nascida prematura } \\
\text { pode apresentar particularidades no contínuo do seu } \\
\text { desenvolvimento, como atrasos. }\end{array}$ & $\begin{array}{l}\text { Lemos e Veríssimo, } 2015^{34} \\
\text { Pal et al., } 2014^{44}\end{array}$ \\
\hline
\end{tabular}

vividos pela criança nos seus primeiros anos, em especial no contexto da família, definem seu bem -estar biopsicossocial e qualidade de vida ${ }^{58}$.

Dessa forma, a força do material educativo consiste em buscar garantir a relação entre as evidências científicas e empíricas e o modelo PPCT, uma vez que teve toda sua elaboração fundamentada nos componentes processo, pessoa, contexto e tempo. Definir tal base teórica é consistente para a finalidade da promoção do desenvolvimento da criança pela família, dado que os processos proximais atuando como motor e forte preditores do desenvolvimento humano podem minimizar ou mesmo deter influências contextuais de vulnerabilidade ${ }^{23}$. O destaque para a força direcionadora dos processos proximais no desenvolvimento da criança também pode ser visualizada no formato de história dialogada com a família, sublinhando as interações entre família e criança no contínuo do desenvolvimento de todas as Pessoas do núcleo familiar.

O formato de história dialogada com o leitor utilizado no material educativo é condizente com o referencial da educação popular e saúde. $\mathrm{O}$ material como ferramenta mediadora já traz em si esta concepção de problematizar com os cui- 
Quadro 3. Aspectos relacionados ao cuidado da criança nascida prematura segundo o desenvolvimento funcional e os interesses da família e suas fontes bibliográficas.

\begin{tabular}{|c|c|c|}
\hline $\begin{array}{c}\text { Classificação } \\
\text { Temática }\end{array}$ & Aspecto do cuidado & Referência \\
\hline \multirow{7}{*}{$\begin{array}{l}\text { Interesses, } \\
\text { dúvidas da } \\
\text { família }\end{array}$} & Desenvolvimento da criança nascida prematura. & Lemos e Veríssimo, $2015^{34}$ \\
\hline & Causas do nascimento prematuro e/ou gravidez de risco. & Lemos e Veríssimo, $2015^{34}$ \\
\hline & Alimentação da criança nascida prematura. & Lemos e Veríssimo, $2015^{34}$ \\
\hline & Conhecimento de testes específicos que a criança precisa realizar. & Lemos e Veríssimo, $2015^{34}$ \\
\hline & Posicionamento e formas de carregar a criança. & Lemos e Veríssimo, $2015^{34}$ \\
\hline & Doenças mais comuns no prematuro. & Lemos e Veríssimo, $2015^{34}$ \\
\hline & Conhecer a experiência de outros cuidadores. & Lemos e Veríssimo, $2015^{34}$ \\
\hline \multirow[t]{4}{*}{$\begin{array}{l}\text { Desenvolvimento } \\
\text { Funcional }\end{array}$} & $\begin{array}{l}\text { O desenvolvimento funcional é considerado consequência } \\
\text { natural e resultado do ensino. }\end{array}$ & Lemos e Veríssimo, $2015^{34}$ \\
\hline & $\begin{array}{l}\text { Prematuros apresentam escores inferiores à população } \\
\text { normativa em testes de avaliação do desempenho funcional e } \\
\text { independência para realização de atividades de vida diária em } \\
\text { três áreas principais do desenvolvimento funcional: autocuidado, } \\
\text { mobilidade e função social mesmo com correção da idade. }\end{array}$ & Lemos e Veríssimo, $2016^{6}$ \\
\hline & $\begin{array}{l}\text { Efeitos cumulativos de vários fatores ambientais e biológicos } \\
\text { influenciam o desenvolvimento funcional de prematuros. }\end{array}$ & Lemos e Veríssimo, $2016^{6}$ \\
\hline & $\begin{array}{l}\text { Prematuros têm um ambiente menos estimulador em } \\
\text { quantidade e qualidade ao desenvolvimento funcional. O } \\
\text { desenvolvimento funcional pode estar relacionado às práticas de } \\
\text { cuidado e ao contexto em que os sujeitos estão inseridos. }\end{array}$ & Lemos e Veríssimo, $2016^{6}$ \\
\hline
\end{tabular}

dadores de nascidos prematuros o cuidado para promoção do desenvolvimento, que vai sendo trabalhado a partir de personagens que vivem experiência similar, buscando uma identificação. O caderno de educação popular e saúde ${ }^{12}$ enfatiza:

A educação popular em saúde não é um processo de transmissão de conhecimento, mas de ampliação dos espaços de interação cultural e negociação entre os diversos atores envolvidos em determinado problema social para a construção compartilhada do conhecimento e da organização politica, necessários a sua superação. Em vez de procurar difundir conceitos e comportamentos considerados corretos, procura problematizar, em uma discussão aberta, o que está incomodando e oprimindo. ${ }^{12}$ (p.21).

$\mathrm{O}$ foco no desenvolvimento funcional como constructo chave do material vem da emergente necessidade em se considerar a Pessoa/criança nascida prematura em seus Contextos e Processos. Apresenta também uma proposta que veicule de alguma maneira o conceito ampliado de saúde ${ }^{59}$ e o modelo conceitual da Classificação Internacional de Funcionalidade $(\mathrm{CIF})^{60}$ que considera os acometimentos da estrutura e função do corpo como componentes de um modelo que estabelece com igual valor aos outros com- ponentes: a atividade, a participação, e os fatores ambientais. Isso tudo está afinado com o modelo bioecológico de desenvolvimento humano ${ }^{23,24,58}$.

A busca de participação e divulgação do material junto ao público alvo durante sua construção remete também ao apelo de colocar as pesquisas acadêmicas como catalisadores de transformações políticas e sociais. Almeja que os participantes de pesquisas transcendam o papel de informantes e receptores de conclusões, efetivando sua participação na produção dos conhecimentos sobre suas singulares realidades ${ }^{22}$. Isso colabora para a consolidação dos Referenciais da Educação Popular ${ }^{12,13}$ e da Teoria Bioecológica ${ }^{23}$ no cerne de pesquisas e práticas de saúde.

\section{Considerações finais}

Este estudo descreve os passos metodológicos para elaboração de um material educativo dirigido à promoção do desenvolvimento da criança nascida prematura partindo da pesquisa participativa com a população alvo, revisão de literatura e referenciais de apoio à construção de materiais educativos. A utilização das múltiplas estratégias 
complementares, focadas na literatura científica e no público alvo, permitiram maior rigor e ampliação do escopo do material educativo. Constituíram-se as estratégias de aproximação com o saber da população, a busca da literatura científica atualizada e busca de literatura de organização de materiais educativos e informativos adequados, como táticas de igual magnitude e importância na elaboração do material educativo, sendo recomendada esta tríplice estratégia para estudos com similar objetivo.

\section{Colaboradores}

Ambas as autoras participaram de todas as etapas de preparação do manuscrito, incluindo discussão dos resultados, revisão e aprovação da versão final do trabalho.

\section{Agradecimentos}

Pelo apoio financeiro recebido da Fundação de Amparo à Pesquisa do Estado de São Paulo FAPESP, e da Coordenação de Aperfeiçoamento de Pessoal de Nível Superior, Brasil - CAPES. 


\section{Referências}

1. Kiney MV, Lawn JE, Howson CP, Belizan J. 15 million preterm births annually: what has changed this year? Reproductive Health 2012; 9:28.

2. World Health Organization (WHO). Born too soon: the global action report on preterm birth. Geneva: WHO; 2012.

3. Victora CG, Aquino EMMLL, Leal MC, Monteiro CA, Barros FCLF, Szwarcwald CL. Saúde de mães e crianças no Brasil: progressos e desafios. Lancet 2011; (Série Brasil):32-46.

4. Picciolini $\mathrm{O}$, Porro $\mathrm{M}$, Meazza $\mathrm{A}$, Giannì $\mathrm{M}$, Rivoli C, Lucco G, Barretta F, Bonzini M, Mosca F. Early exposure to maternal voice: Effects on preterm infants development. Early Human Development 2014; 90(6):287-292.

5. Lemos RA, Fronio JS, Ribeiro LC, Demarchi R, Silva J, Neves LAT. Functional performance according to gestational age and birth weight of preschool children born premature or with low weight. Rev Bras Cresc Desenvolv Human 2012; 22:17-26.

6. Lemos RA, Veríssimo MLOR. Functional development of preterm infants: an integrative literature review. Fisioter Mov 2016; 29(3):623-633.

7. Moreira RS, Magalhães LC, Alves CRL. Effect of preterm birth on motor development, behavior, and school performance of school-age children: A systematic review. J Pediatr (Rio J) 2014; 90(2):119-134.

8. Brasil. Ministério da Saúde (MS), Secretaria de Atenção à Saúde, Departamento de Ações Programáticas e Estratégicas. Atenção à saúde do recém-nascido: guia para os profissionais de saúde. Brasília: MS; 2011.

9. Chiodi LC, Aredes ND, Scochi CGS, Fonseca LMM Educação em saúde e a família do bebê prematuro: uma revisão integrativa. Acta Paul Enf 2012; 25(6):969-974.

10. Couto FF, Praça NS. Preparo dos pais de recém-nascido prematuro para alta hospitalar: uma revisão bibliográfica. Rev Esc Enferm USP 2009; 13(4):886-891.

11. Brasil. Ministério da Saúde (MS). Gabinete do Ministro. Portaria no 2.761, de 19 de novembro de 2013. Institui a Política Nacional de Educação Popular em Saúde no Âmbito do Sistema Único de Saúde (PNEPS-SUS). Diário Oficial da União; 2013.

12. Brasil. Ministério da Saúde (MS). Secretaria de Gestão Estratégica e Participativa. Departamento de Apoio à Gestão Participativa. Caderno de educação popular e saúde/Ministério da Saúde, Secretaria de Gestão Estratégica e Participativa, Departamento de Apoio à Gestão Participativa. Brasília: MS; 2007.

13. Brasil. Ministério da Saúde (MS). Secretaria de Gestão Estratégica e Participativa. Departamento de Apoio à Gestão Participativa. II Caderno de educação popular e saúde/Ministério da Saúde, Secretaria de Gestão Estratégica e Participativa, Departamento de Apoio à Gestão Participativa. Brasília: MS, 2014.

14. Silva EP, Lima RT, Osório MM. Impacto de estratégias educacionais no pré-natal de baixo risco: revisão sistemática de ensaios clínicos randomizados. Cien Saude Colet 2016; 21(9):2935-2948.

15. Santana MCCP, Goulart BNG, Chiari BM, Melo AM, Silva EHAA. Aleitamento materno em prematuros: atuação fonoaudiológica baseada nos pressupostos da educação para promoção da saúde. Cien Saude Colet 2010; 15(2):411-417.
16. Merhy EE, Onocko R, organizadores. Agir em saúde: um desafio para o público. $2^{\text {a }}$ ed. São Paulo: Hucitec; 2002.

17. Alexandre NMC, Coluci MZO. Content validity in the development and adaptation processes of measurement instruments. Cien Saude Colet 2011; 16(7):30613068 .

18. Coluci MZO, Alexandre NMC, Milani D. Construção de instrumentos de medida na área da saúde. Cien Saude Colet 2015; 20(3):925-936.

19. Moreira RS, Figueiredo EM. Instruments of assessment for first two years of life of infant; J Human Grow Development 2013; 23(2):215-221.

20. Almeida ER, Moutinho CB, Leite MTS. Family health nurses' teaching practice in the health education development. Interface (Botucatu) 2016; 20(57):389-401.

21. Silva EP, Lima RT, Osório MM. Impacto de estratégias educacionais no pré-natal de baixo risco: revisão sistemática de ensaios clínicos randomizados. Cien Saude Colet 2016; 21(9):2935-2948.

22. Streck DR. Participatory research methodologies and popular education: reflections on quality criteria. Interface (Botucatu) 2016; 20(58):537-547.

23. Bronfrenbrenner U. Bioecologia do desenvolvimento humano: tornando os seres humanos mais humanos. Porto Alegre: Artmed; 2011.

24. Tudge J. A teoria de Urie Bronfenbrenner: Uma teoria contextualista? [texto na Internet]. 2008 [acesso 2018 Jan 24]. Disponível em: http://www.uncg.edu/hdf/ facultystaff/Tudge/Tudge,\%202008.pdf

25. Souza MT, Silva MD, Carvalho R. Revisão integrativa: o que é e como fazer. Einstein 2010; 8(1 Pt. 1):102-106.

26. US Centers for Disease Control and Prevention (CDC). Simply Put: A guide for creating easy-to-understand materials. Atlanta: CDC; 2009.

27. Msall ME, Avery RC, Tremont MR, Lima JC, Rogers ML, Hogan DP. Functional disability and school activity limitations in 41,300 school-age children: relationship to medical impairments. Pediatrics 2003; 111(3):548-553.

28. Mancini MC. Inventário de Avaliação Pediátrica de Incapacidade (PEDI). Belo Horizonte: Editora UFMG; 2005.

29. Reberte LM, Hoga LAK, Gomes ALZ. O processo de construção de material educativo para a promoção da saúde da gestante. Rev Lat Am Enfermagem 2012; 20(1):101-108.

30. Queiroz MVO, Dantas MCQ, Ramos IC, Jorge MSB. Tecnologia do cuidado ao paciente renal crônico: enfoque educativo-terapêutico a partir das necessidades dos sujeitos. Texto Contexto Enferm 2008; 17(1):55-63.

31. Teixeira E, Siqueira AA, Silva JP, Lavor LC. Cuidados com a saúde da criança e validação de uma tecnologia educativa para famílias ribeirinhas. Rev Bras Enferm 2011; 64(6):1003-1009.

32. Liévano-Fiesco M, García-Londoño G, Leclercq-Barriga M, Liévano-De Lombo G, Solano-Salazar K. Validación del material lúdico de la estrategia educativa basada em juegos para la promoción de estilos de vida saludable en niños de cuatro a cinco años de edad. Universitas Scientiarum 2009; 14(1):79-85. 
33. Fonseca LMM, Scochi CGS, Rocha SMM, Leite AM. Cartilha educativa para orientação materna sobre os cuidados com o bebê prematuro. Rev Lat Am Enfermagem 2004; 12(1):65-75.

34. Lemos RA, Veríssimo MLOR. Development of premature children: caregivers'understanding according to the Bioecological Theory. Rev Esc Enferm USP 2015; 49(6):898-906.

35. Lundqvist P, Hellström-Westas L, Hallström I. Reorganizing Life: A Qualitative Study of Fathers' Lived Experience in the 3 Years Subsequent to the Very Preterm Birth of Their Child. J Pediatric Nursing 2014; 29:124-131.

36. Hall EOC, Kronborg H, Aagaard H, Brinchmann BS. The journey towards motherhood after a very preterm birth: Mothers' experiences in hospital and after home-coming. J Neonatal Nursing 2013; 19:109e113.

37. Evans T, Whittingham K, Sanders M, Colditz P, Boyd RN. Development are parenting interventions effective in improving the relationship between mothers and their preterm infants? Infant Behavior Development 2014; 37:131-154.

38. Caskey M, Stephens B, Tucker R, Vohr B. Importance of Parent Talk on the Development of Preterm Infant Vocalizations. Pediatrics 2011; 128(5):e1-e8.

39. Gerstein ED, Poehlmann-Tynan J, Clark R. Mother-Child Interactions in the NICU: Relevance and Implications for Later Parenting. J Pediatric Psychology 2015; 40(1):33-40.

40. Gabis LV, Hacham-Pilosof KOT, Yosef OB, Rabinovitz G3, Leshem G, Shilon-Hadass A, Biran Y, Reichman B, Kuint J, Bart O. The Influence of a Multisensory Intervention for Preterm Infants Provided by Parents, on Developmental Abilities and on parental Stress Levels. J Child Neurol 2015; 30(7):896-903.

41. Whittingham K, Boyd RN, Sanders MR, Colditz P. Parenting and Prematurity: Understanding Parent Experience and Preferences for Support. J Child Fam Stud 2014; 23:1050-1061.

42. Morais AC, Quirino MD, Almeida MS. O cuidado da criança prematura no domicílio. Acta Paul Enferm 2009; 22(1):24-30.

43. McGowan JE, Alderdice FA, Boylan J, Holmes VA, Jenkins J, Craig S, Perra O, Johnston L. Neonatal intensive care and late preterm infants: Health and Family functioning at three years. Early Human Development 2014; 90:201-205.

44. Pal van der SM, Alpay LL, Steenbrugge GJ, Detmar SB. An Exploration of Parents' Experiences and Empowerment in the Care for Preterm Born Children. J Child Fam Stud 2014; 23:1081-1089.

45. Winstanley A, Sperotto RG, Putnick DL, Cheriand $\mathrm{S}$, Bornstein MH, Gattis M. Consistency of maternal cognitions and principles across the first five months following preterm and term deliveries. Infant Behavior Development 2014; 37:760-771.

46. Howe TH, Sheu CF, Wang TN, Hsu YW. Parenting stress in families with very low birth weight preterm infants in early infancy. Res Dev Disabil 2014; 35(7):1748-1756.

47. Gray PH, Edwards DM, O'Callaghan MJ, Cuskelly M, Gibbons K. Parenting stress in mothers of very preterm infants - Influence of development, temperament and maternal depression. Early Hum Dev 2013; 89(9):625-629.
48. Chang HP, Chen JY, Huang YH, Tyan JY, Yeh CJ, Su $\mathrm{PH}$, Chen VCH. Prevalence and Factors Associated with Depressive Symptoms in Mothers with Infants or Toddlers. Pediatr Neonatol 2014; 55:470e479.

49. Mehler K, Mainusch A, Hucklenbruch-Rother E, Hahn M, Hünseler C, Kribs A. Increased rate of parental postpartum depression and traumatization in moderate and late preterm infants is independent of the infant's motor repertoire. Early Hum Dev 2014; 90(12):797-801.

50. Suttora C, Spinelli M, Monzani D. From prematurity to parenting stress: The mediating role of perinatal post-traumatic stress disorder. Eur J Dev Psychol 2013; 11(4):478-493.

51. Huhtala M, Korja R, Lehtonen L, Haataja L, Lapinleimu H, Rautava P. Associations between parental psychological well-being and socio-emotional development in 5-year-old preterm children. Early Hum Dev 2014; 90: 119-124.

52. McManusa BM, Poehlmannb J. Parent-child interaction, maternal depressive symptoms and preterm infant cognitive function. Infant Behav Dev 2012; 35(3):489-498.

53. Taubman O, Ben-Ari, Spielman V. Personal Growth Following the First Child's Birth: A Comparison of Parents of Pre- and Full-Term Babies. Social Work Research 2014; 38(2):91-106.

54. Costa SAF, Ribeiro CA, Borba RIH, Balieiro MMFG. A experiência da família ao interagir com o recém nascido prematuro no domicílio. Esc Anna Nery Rev Enferm 2009; 13(4):741-749.

55. Raffray M, Semenic S, Galeano SO, Marín SCO. Barriers and facilitators to preparing families with premature infants for discharge home from the neonatal unit. Perceptions of health care providers. Invest Educ Enferm 2014; 32(3):379-392.

56. Guillaume S, Michelin N, Amrani E, Benier B, Durrmeyer X, Lescure S, Bony C, Danan C, Baud O, Jarreau PH, Zana-Taïeb E, Caeymaex L. Parents' expectations of staff in the early bonding process with their premature babies in the intensive care setting: a qualitative multicenter study with 60 parents. $B M C$ Pediatrics 2013; 13:18.

57. Custódio ZAO, Crepaldi MA, Linhares MBM. Redes sociais de apoio no contexto da prematuridade: perspectiva do modelo bioecológico do desenvolvimento humano. Estudos Psicologia 2014; 31(2):247-255.

58. Benetti IC, Vieira ML, Crepaldi MA, Schneider DR Fundamentos da teoria bioecológica de Urie Bronfenbrenner. Pensando Psicol 2013; 9(16):89-99.

59. Brasil. Lei no 8.080 , de 19 de Setembro de 1990. Dispõe sobre as condições para a promoção, proteção e recuperação da saúde, a organização e o funcionamento dos serviços correspondentes e dá outras providências. Diário Oficial da União 1990; 20 set.

60. World Health Organization (WHO). International Classification of functioning, disability and health (ICF). Geneva: WHO; 2001.

Artigo apresentado em 16/02/2018

Aprovado em 18/06/2018

Versão final apresentada em 20/06/2018 